\title{
Structure and random anisotropy in single-phase Ni nanocrystals
}

\author{
Eiji Kita, ${ }^{\text {a) }}$ Naoki Tsukuhara, Hidenori Sato, Keishin Ota, ${ }^{\text {b) }}$ and Hideto Yangaihara \\ Institute of Applied Physics, University of Tsukuba, Tsukuba, Ibaraki 305-8573, Japan \\ Hisanori Tanimoto \\ Institute of Materials Science, University of Tsukuba, Tsukuba, Ibaraki 305-8573, Japan \\ Naoshi Ikeda \\ JASRI, Kouto, Mikazuki-cho Sayo-gun, Hyogo 679-5198, Japan
}

(Received 15 July 2005; accepted 23 February 2006; published online 10 April 2006)

\begin{abstract}
We prepared high-purity oxygen-free and single-phase Ni nanocrystals using the gas condensation and deposition method in order to examine the random anisotropy model (RAM). The grain size of an as-prepared sample was estimated to be $8-10 \mathrm{~nm}$. Thermal annealing increased grain sizes $(D)$ up to $22 \mathrm{~nm}$. Clear $D^{6}$ dependence of the coercive force on a grain size smaller than $13 \mathrm{~nm}$ was obtained for samples prepared on both plastic and $\mathrm{KBr}$ single-crystal substrates. For larger grain sizes, $H_{\mathrm{c}}$ decreased with $D^{-1}$ and these results clearly conform to the RAM in single-element nanocrystal systems. A local anisotropy constant was estimated from the slope of the $D^{6}$ relation and the peak of $H_{\mathrm{c}}$ at $D=13 \mathrm{~nm}$. (C) 2006 American Institute of Physics. [DOI: 10.1063/1.2188597]
\end{abstract}

Ferromagnetic nanocrystalline materials have been investigated since the discovery of excellent soft magnetic properties in thermally recrystallized amorphous materials. ${ }^{1,2}$ The steep decrease in coercive force was interpreted by Herzer using the random anisotropy model (RAM). ${ }^{3}$ This model clearly supports the theorem that the coercive force $\left(H_{c}\right)$ is proportional to $D^{6}$ of the grain size $(D)$ when the magnetic anisotropy over the magnetically coherent area that covers a large number of crystallites is averaged.

The original model was created for use in a simple system but has been extended to systems with two separate phases and systems accompanied by uniform uniaxial magnetic anisotropy. ${ }^{4}$ Those are more realistic, in practical materials made those of amorphous states. ${ }^{5}$ To understand the role of averaging of crystalline anisotropy, experimental evidence on single element and single phase nanocrystals is required. Fe and $\mathrm{Ni}$ nanocrystalline materials have been reported as being prepared using a gas condensation and compaction technique. ${ }^{6}$ However, the results on the systems were not well supported by the simple RAM.

A gas condensation and deposition method (GDM) (Ref. 7) has been developed for the production of nanocrystalline materials. ${ }^{8}$ In the GDM, a simple process prevents the surface oxidization of ultra-fine particles (UFPs) and oxygenfree metallic nanocrystals can be produced. The density of the GDM nanocrystals was reported almost the same as the bulk for the Au nanocrystals ${ }^{9}$ and the porosity of the GDM nanocrystals is considered to be the same order or better than those from other techniques. In this letter, we demonstrate the $D^{6}$ dependence of coercive force on the grain size of single phase Ni nanocrystals $(n-\mathrm{Ni})$ prepared using GDM. Additionally, the structure and magnetic anisotropy that appeared in the nanocrystals are discussed.

$\mathrm{Ni}$ nanocrystals were produced using the GDM. ${ }^{8}$ UFPs were prepared by heating $\mathrm{Ni}$ metal in a carbon crucible in an evaporation chamber keeping the $\mathrm{He}$ gas pressure at $0.1 \mathrm{MPa}$

\footnotetext{
${ }^{a)}$ Electronic mail: kita@bk.tsukuba.ac.jp

${ }^{b}$ Present address: Microphase Co. Ltd., Tokodai 5-9-1, Tsukuba, Ibaraki 300-2635, Japan.
}

(760 Torr) similar to the manner used for inert gas evaporation. ${ }^{10}$ The UFPs were transferred into a deposition chamber because of the He gas flow resulting from the pressure difference and were deposited on a substrate using a nozzle with a $0.6 \mathrm{~mm}$ inner diameter. In this process, nanocrystals are formed within a few milliseconds after generating UFP and the oxidization can be suppressed. Polyimide (Kapton) films and $\mathrm{KBr}$ single-crystal wafers were used as substrates. The nanocrystals were several $\mu \mathrm{m}$ thick, much larger than the coherent length of the magnetic exchange coupling.

$\mathrm{X}$-ray diffraction (XRD) studies were carried out in two ways. First, high-energy (25 keV) XRD study using a synchrotron orbital radiation X-ray source at SPring-8, BL2B1, was performed on a limited number of samples to obtain higher-order diffraction peaks and high-quality data. Measurements were carried out for reflection and transmission geometries to check the anisotropic alignment of crystal axes. Second, for laboratory XRD measurement, a conventional diffractometer (Rigaku RINT 2000) with $\mathrm{Cu} K \alpha$ radiation was used. Both data sets for the same samples were compared to estimate the grain sizes using Scherrer's equation and a Wilson plot. The oxygen content was confirmed to be less than 5\% with Auger electron spectroscopy (AES). Magnetization curves were obtained using a vibrating sample magnetometer. Thermal annealing was carried out in a vacuum to increase grain sizes.

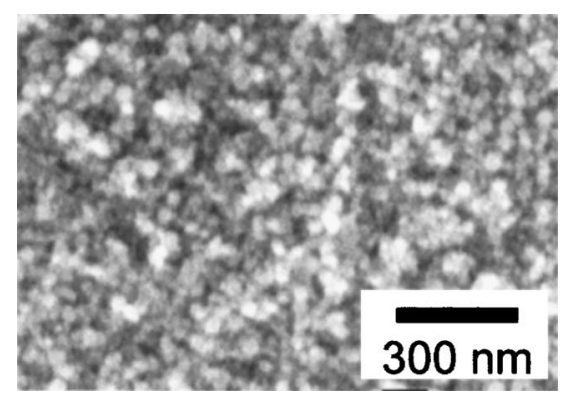

FIG. 1. SEM image of Ni nanocrystals prepared with GDM. 


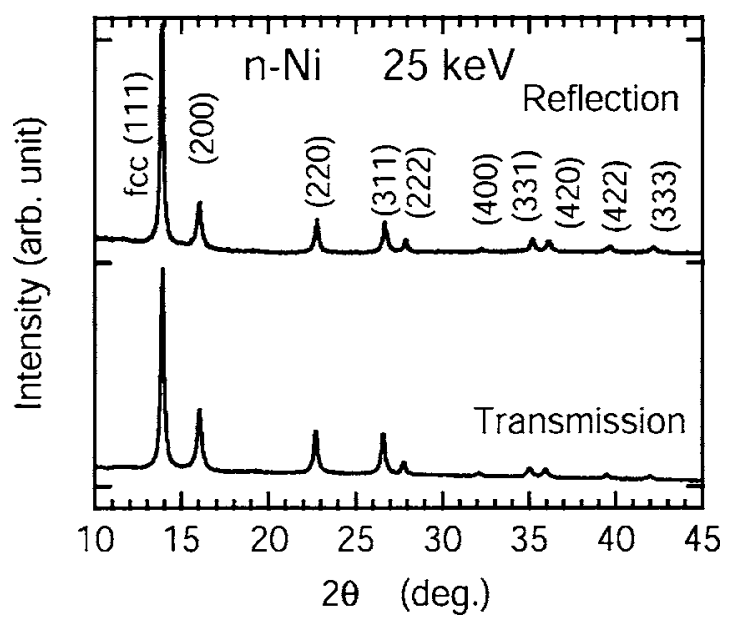

FIG. 2. XRD patterns of the $n$-Ni prepared with GDM. Data were taken at an $\mathrm{x}$ ray of energy $25 \mathrm{keV}$.

A scanning electron microscope (SEM) image of $\mathrm{Ni}$ nanocrystals is shown in Fig. 1. Small grains with a size of $10 \mathrm{~nm}$ are seen. However, it is difficult to ascertain the change in average sizes after thermal annealing. Figure 2 shows the XRD patterns of $n$-Ni annealed at $350{ }^{\circ} \mathrm{C}$ for $10 \mathrm{~min}$. More than nine reflections from the face-centeredcubic (fcc) Ni were detected and no remarkable difference was seen in the profiles obtained with reflection and transmission geometries. It is noted that significant $\langle 111\rangle$ preferred orientation normal to the substrate has been found in Au nanocrystals prepared in the same manner. ${ }^{9}$ The Wilson plot of the reflection geometry data is shown in Fig. 3. The solid line represents the results of the least-squares fitting. The slope and the $y$-axis intercept lead to a mean diameter of $10.8 \mathrm{~nm}$ and nonuniform strain of $0.16 \%$, respectively. The points for two low angle diffractions deviated from the fitted line. However, the other seven points can be fitted with a linear line given by a fitting for all points. A similar trend has been attributed to stacking faults on the fcc (111) plane. ${ }^{11}$ This narrow linewidth may be attributed to the twinned structure, where two individual fine particles possess (111) planes. In such a case, only (111) reflections have a narrow linewidth. It is noted that multiple twinned grains, in which adjacent grains share (111) planes, were often found in ultrafine particles of fcc metals. ${ }^{12}$

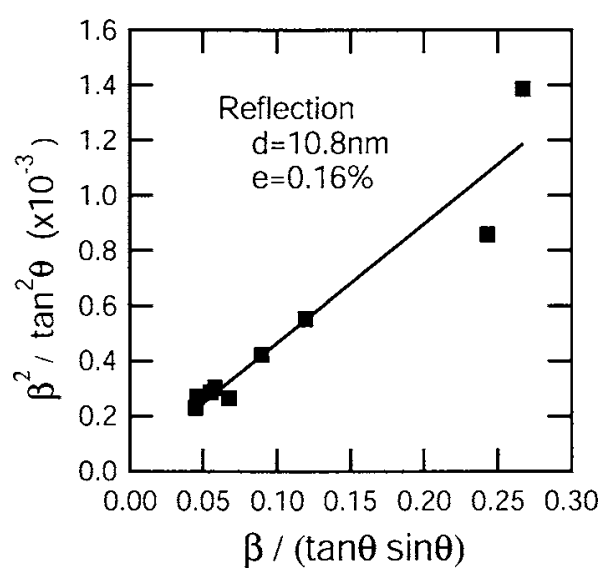

FIG. 3. Wilson plot for the $n$-Ni prepared with GDM. The reflection data

mechanism is that the exchange stiffness is weakened by 0.5
from Fig. 2 was used for plotting.
Downloaded 03 Apr 2007 to 130.158 .56 .189 . Redistribution subject to AlP license or copyright, see http://apl.aip.org/apl/copyright.jsp

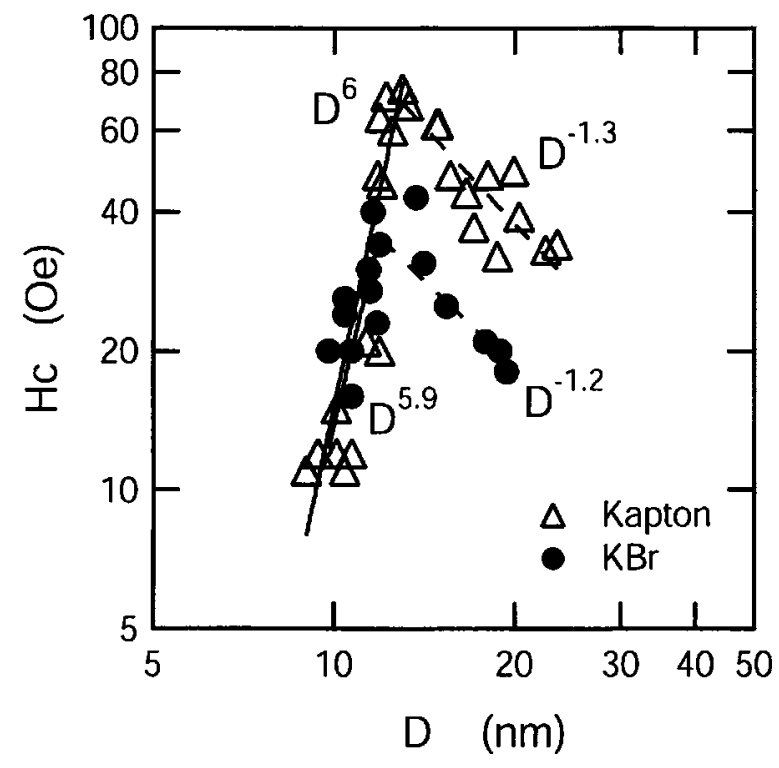

FIG. 4. Grain size $(D)$ dependence of coercive force $\left(H_{c}\right)$ for $n$-Ni. Data for $n$-Ni prepared on Kapton substrates $(\triangle)$ and the $\mathrm{KBr}$ substrates $(\bullet)$ were shown.

Data from the laboratory experiments well reproduce the low angle part of the Wilson plot. The diameter deduced from the plot was compared to the averaged value of the diameters of four peaks given by Scherrer's relation and these amplitudes were almost equal. We used this averaged size estimated from the laboratory experiments as a true grain size.

The grain size increased to $20 \mathrm{~nm}$ when the sample was annealed at $400{ }^{\circ} \mathrm{C}$. Coercive forces are plotted against the mean grain size of the Ni nanocrystals in Fig. 4. For two samples prepared on different substrates, $H c$ 's increase steeply and then decrease moderately as the grain sizes increase. Solid lines in Fig. 4 are results of fitting for the nanocrystalline region and the multidomain region. The numbers of the power obtained were $6.0 \pm 1.1$ and 5.9 for polyimide and $\mathrm{KBr}$ substrates, respectively. The latter value includes a much larger margin of error than that for the polyimide substrate samples due to the narrow range of particle diameter. However, it agrees with the theoretically predicted value of $6 .^{3}$

Local magnetic anisotropy can be evaluated from the RAM relation, where $H_{c}$ is given by the following equation,

$$
H_{c}=P_{c} \frac{K_{1}}{M_{s}}\left(\frac{D}{L_{\mathrm{ex}}}\right)^{3 / 2}=P_{c} \frac{K_{1}^{4} D^{6}}{M_{s} A^{3}},
$$

where $K_{1}, M_{s}, A$, and $P_{c}$ are a local anisotropy constant, saturation magnetization, an exchange stiffness constant, and a constant factor ranging between 0.2 and 2 (Ref. 3), respectively. $L_{\text {ex }}$ is a correlation length defined as $\varphi \sqrt{A /\left\langle K_{1}\right\rangle}$ with $\varphi=1$. In spite of the fact that the $n$-Ni exhibits sixth-power behavior, ${ }^{13}$ the absolute value of $H_{c}$ is much larger than the calculated value using the bulk value of magnetic anisotropy. There are two interpretations to explain this enhancement. The first one is that the local magnetic anisotropy $K_{1}$ is solely enhanced to be $2.8 \pm 0.8 \times 10^{5} \mathrm{erg} / \mathrm{cm}^{3}$ from that of bulk, $5.7 \times 10^{4} \mathrm{erg} / \mathrm{cm}^{3}$ (Ref. 14) without changing other parameters. This enhancement can be attributed to the surface magnetic anisotropy that is reported on the UFP. ${ }^{15}$ The other mechanism is that the exchange stiffness is weakened by 0.5 Downloaded 03 Apr 2007 to 130.158.56.189. Redistribution subject to AIP license or copyright, see http://apl.aip.org/apl/copyright.jsp 


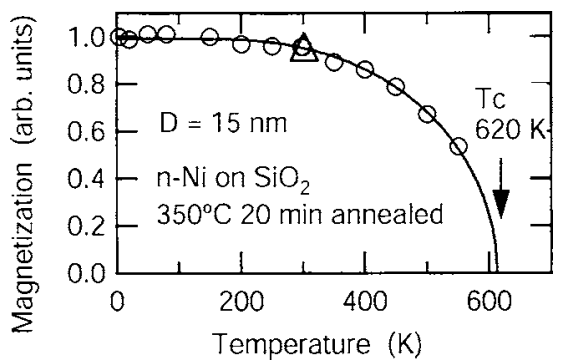

FIG. 5. Temperature dependence of magnetization for $n$-Ni prepared on an $\mathrm{SiO}_{2}$ substrate. A solid line indicates the result of fitting using Brillouin function with $S=1 / 2 . T_{C}$ was estimated to be $620 \mathrm{~K}$. A triangle $(\triangle)$ depicts the room temperature amplitude after the higher temperature measurement.

times. However, no remarkable reduction of the magnetization was detected for annealed samples below the annealing temperature (see Fig. 5). Therefore, enhancement by the local anisotropy is realistic. Such enhancement in fine particles has been sometimes reported and the factor of 5 in this case can be attributed to that mechanism. The structural model derived from the powder XRD profile proposed as that random chain of grains connected with (111) planes also supports the averaging of anisotropy.

The peak value of $H_{c}(=76 \mathrm{Oe})$ was placed at $D$ $=13 \mathrm{~nm}$. In the RAM argument, ${ }^{16}$ this size was equal to the basic exchange length $L_{\mathrm{ex}}^{0}=\varphi \sqrt{A / K_{1}}$, where $\varphi$ ia a proportionality factor in the order of one and $P_{c}$ in Eq. (1) is replaced by $P_{c} / \varphi^{6}$. Using the relation, $H_{c}=P_{c} K_{1} / M_{s}$ at the peak and assuming $P_{c}=0.64, K_{1}$ was obtained to be 5.7 $\times 10^{4} \mathrm{erg} / \mathrm{cm}^{3}$, which is close to the bulk Ni anisotropy constant. The result leads to $\varphi=0.36$ using $A=0.75$ $\times 10^{-6} \mathrm{erg} / \mathrm{cm}^{14}$ This analysis enables us to estimate the local magnetic anisotropy $\left(K_{1}\right)$ in the $n$-Ni and $\varphi$ with only one open parameter, $P_{c}$, however, the amplitude of $\varphi$ is smaller than the expected value of 1.5 (Ref. 16). This is another qualitatively reasonable interpretation of the position of the peak. The difference in the positions of the peaks between the different substrates may be due to stress caused by the difference in the thermal expansion coefficients of the substrate and nanocrystals.
We reported that high-energy XRD was effective in determining grain size. Clear $D^{6}$ dependence in the relation between grain size $(D)$ and coercive force $\left(H_{c}\right)$ was demonstrated in single-phase Ni nanocrystals. The magnetic anisotropy constant was estimated from the slope of the $D^{6}$ relation and the peak position.

The authors would like to thank Dr. H. Murakami for his help in AES and SEM measurements. This work was supported by the Center for Tsukuba Advanced Research Alliance (TARA) at the University of Tsukuba. The XRD study at SPring-8 was carried out under Contract No. 2002A0638. This work has been partly supported by the 21 st Century COE program, "Promotion of Creative Interdisciplinary Materials Science for Novel Functions" under MEXT $<$ Japan and by the University of Tsukuba Nanoscience Special Project.

${ }^{1}$ Y. Yoshizawa, S. Ogawa, and K. Yamauchi, J. Appl. Phys. 64, 6044 (1988).

${ }^{2}$ K. Suzuki, A. Makino, A. Inoue, and T. Masumoto, J. Appl. Phys. 70, 6232 (1991)

${ }^{3}$ G. Herzer, IEEE Trans. Magn. 26, 1397 (1990).

${ }^{4}$ G. Herzer, Scr. Metall. Mater. 33, 1741 (1995).

${ }^{5}$ K. Suzuki and J. Cadogan, Phys. Rev. B 58, 2730 (1999).

${ }^{6}$ J. F. Löffler, J. Meier, B. Doudin, J.-P. Ansermet, and W. Wagner, Phys. Rev. B 57, 2915 (1998).

${ }^{7}$ S. Kashu, E. Fuchita, T. Manabe, and C. Hayashi, Jpn. J. Appl. Phys., Part 2 23, L910 (1984).

${ }^{8}$ Y. Sasaki, M. Hyakkai, E. Kita, A. Tanimoto, A. Tasaki, and Y. Iwamoto, J. Appl. Phys. 81, 4736 (1997).

${ }^{9}$ S. Sakai, H. Tanimoto, E. Kita, and H. Mizubayashi, Phys. Rev. B 66 214106 (2002).

${ }^{10}$ R. Uyeda, Prog. Mater. Sci. 35, 1 (1991).

${ }^{11}$ B. E. Warren, X-ray Diffraction (Addison-Wesley, Reading, MA, 1969) Chap. 13, p. 275.

${ }^{12}$ S. Ino, J. Phys. Soc. Jpn. 32, 999 (1964).

${ }^{13}$ T. Sakai, N. Tsukahara, H. Tanimoto, K. Ota, H. Murakami, and E. Kita, Scr. Mater. 44, 1359 (2001).

${ }^{14}$ S. Chikazumi, Physics of Ferromagnetism (Oxford University Press, Oxford, UK, 1997).

${ }^{15}$ V. Dupuis, J. Perez, J. Tuaillon, V. Paillard, P. Melinon, A. Perez, B Barbara, L. Thomas, S. Fayeulle, and J. Gay, J. Appl. Phys. 76, 6678 (1994).

${ }^{16}$ G. Herzer, J. Magn. Magn. Mater. 294, 99 (2005). 